\title{
PESQUISA EM TEMPOS DE COVID-19: ESTRATÉGIA PARA 0 PLANEJAMENTO EM SAÚDE
}

RESEARCH IN TIMES OF COVID-19: STRATEGY FOR HEALTH PLANNING INVESTIGACIÓN EN TIEMPOS DE COVID-19: ESTRATEGIA PARA EL PLANEAMIENTO EN SALUD

Maria Socorro de Araújo Dias ${ }^{1}$ Verena Emmanuelle Soares Ferreira ${ }^{2}$

Rogeriany Lopes Farias ${ }^{3}$

Lielma Carla Chagas da Silva ${ }^{4}$

Heliandra Linhares Aragão ${ }^{5}$

Rafaela Costa Porto 6

Palavras-chave:

Pesquisa em Sistema de Saúde Pública; Pandemias; Infecção por coronavírus.

Keywords: Research in Public Health System; Pandemics; Coronavirus Infection

Palabras clave: Investigación en Sistema de Salud Pública; Pandemia; Infección por Coronavirus.

Submetido: $07 / 11 / 20$

Aprovado: $27 / 11 / 20$

Autor(a) para Correspondência: Maria Socorro de Araújo Dias Rua Maestro José Pedro, 170 - Centro Sobral-CE CEP: 62010-260 E-mail:socorroad@gmail.com

\section{RESUMO}

A produção científica se constitui como uma ferramenta estratégica para responder a vazios de conhecimento relacionados à pandemia do novo coronavírus. Nesse sentido, este estudo objetiva descrever duas pesquisas que colaboraram com subsidios para a indução de decisões em saúde no curso da pandemia de Covid-19. Trata-se de estudo exploratório descritivo, do tipo relato de experiência, com abordagem qualitativa, realizado em Sobral-CE, de junho a agosto de 2020, período no qual o município foi cenário de investigação para duas pesquisas: "Evolução e Prevalência de infecção por Covid-19 no Brasil" e "Pesquisa de soroprevalência da Covid-19 em Sobral-CE". Ambas contaram com o forte apoio da gestão municipal, do planejamento à execução. Realça-se que o acompanhamento sistemático permitiu que, já no curso do desenvolvimento, as informações coletadas fossem submetidas ao núcleo gestor da Secretaria da Saúde de Sobral para apoiar e/ou redirecionar tomadas de decisões, particularmente no que concerne às intervenções epidemiológicas e sanitárias. A experiência realça a relevância da ciência na produção de evidência e na indução da tomada de decisão em saúde.

1. Enfermeira. Doutora em enfermagem (UFC). Docente da Universidade Estadual Vale do Acaraú (UVA). Diretora da Escola de Saúde Pública Visconde de Saboia (ESP-VS). E-mail: socorroad@gmail.com ORCID: https://orcid. org/0000-0002-7813-547X

2. Enfermeira. Mestre em Saúde da Família (UFC). Gerente da Célula de Vigilância Sanitária. Secretaria da Saúde de Sobral. Prefeitura de Sobral. E-mail: verenaemmanuelle@gmail.com. ORCID: https://orcid.org/0000-0002-3714$\underline{3406}$

3. Assistente Social. Mestranda em Saúde da Família (UVA/Renasf). Gerente da Atenção Primária à Saúde de Sobral. Secretaria da Saúde de Sobral. Prefeitura de Sobral. E-mail: rogerianylopes@gmail.com. ORCID: https://orcid. org/0000-0003-1040-727X

4. Enfermeira. Mestre em Saúde da Família (UFC). Gerente de Pesquisa da Saúde Pública Visconde de Saboia (ESPVS). Secretaria da Saúde de Sobral. Prefeitura de Sobral. E-mail: lielma_carla@yahoo.com.br ORCID: https://orcid. org/0000-0002-2688-9309

5. Assistente Social. Mestranda em Saúde da Família (UFC). Gerente do Centro de Atenção Psicossocial Álcool e outras Drogas. Secretaria da Saúde de Sobral. Prefeitura de Sobral. E-mail: helaindrabj@hotmail.com 0RCID: https://orcid.org/0000-0001-6881-7250

6. Educadora Física. Especialista em Saúde da Família. Gerente do Centro de Reabilitação. Secretaria da Saúde de Sobral. Prefeitura de Sobral. E-mail: rafaelacporto@hotmail.com. ORCID: https://orcid.org/0000-0001-7913-8130 


\section{ABSTRACT}

Scientific production is a strategic tool to respond to knowledge gaps related to the pandemics of the new coronavirus. Thus, this study aims to describe two studies that collaborated with subsidies for the induction of health decisions in the course of the pandemics of Covid-19. A descriptive exploratory study, of the experience report type, with a qualitative approach, carried out in Sobral-CE, from June to August 2020, a period in which this municipality was the investigation scenario for two researches: "Evolução e Prevalência de infecção por Covid-19 no Brasil" (Evolution and prevalence of Covid-19 infection in Brazil) and "Pesquisa de soroprevalência da Covid-19 em Sobral-CE" (Covid-19 seroprevalence survey in Sobral-CE). Both had the strong support from the municipal management, from planning to execution. We emphasize that the systematic monitoring allowed that, already in the development courses, the collected information was submitted to the management core of Sobral Health Department to support and/or redirect decision making, particularly with regard to epidemiological and health interventions. The experience highlights the relevance of science in producing evidence and inducing health decision making.

\section{RESUMEN}

La producción científica se constituye herramienta estratégica para responder a vacíos de conocimiento relacionados a la pandemia del nuevo coronavirus. En ese sentido, este estudio tiene como objetivo describir dos investigaciones que colaboraron con subsidios para la inducción de decisiones en salud en el curso de la pandemia de Covid-19. Estudio exploratorio descriptivo, del tipo relato de experiencia, con enfoque cualitativo, realizado en Sobral-CE, de junio hasta agosto de 2020, periodo ese, en lo que el municipio fue escenario de búsqueda para dos investigaciones: "Evolução e Prevalência de infecção por Covid-19 no Brasil" (Evolución y prevalencia de infección por Covid-19 en Brasil) y "Pesquisa de soroprevalência da Covid-19 em Sobral-CE" (Investigación de soroprevalencia de la Covid-19 en Sobral-CE). Ambas contaron con el fuerte apoyo de la gestión municipal, del planeamiento a la ejecución. Se destaca que el acompañamiento sistemático ha permitido que, en los cursos de desarrollo, las informaciones recogidas fueran sometidas al núcleo de gestión de la Secretaria de la Salud de Sobral para apoyar y redireccionar tomadas de decisiones, particularmente en lo que concierne a las intervenciones epidemiológicas y sanitarias. La experiencia destaca la relevancia de la ciencia en la producción de evidencia e inducción de la tomada de decisión en salud.

\section{INTRODUÇÃO}

A ciência visa conhecer e descrever os fenômenos que ocorrem em nosso meio, ao passo que propõe teorias racionais que explicam por que e como esses fenômenos acontecem ${ }^{1}$. É a ciência indutora de múltiplos processos cotidianos. Sua influência, nem sempre percebida, traduz-se na geração de conhecimentos para o desenvolvimento da humanidade com evidente importância nos diversos meios da atividade humana. Sua necessidade vem sendo reconhecida tanto para a tomada de decisão como para o exercício da plena cidadania, que é fortalecida pelo acesso democrático às informações ${ }^{2}$.

Desde a década de 90, o debate sobre o papel da pesquisa para os sistemas e serviços de saúde se intensificou. No campo internacional, destacaramse atores como o Council on Health Research for Development (COHRED) e o Global Forum for Health Research que, de forma complementar, buscam fortalecer a pesquisa em saúde nas agendas nacionais, regionais e global, na perspectiva de promover 0 desenvolvimento e reduzir as iniquidades em saúde. Ademais, o uso do conhecimento científico para a tomada de decisão tem se tornado uma prática comum. $\mathrm{Na}$ área da saúde, existe um crescente interesse, em todo o mundo, em garantir a elaboração de políticas públicas informadas por evidência, o que pode possibilitar a melhoria do desempenho do sistema de saúde, além de evitar iniquidades provenientes de políticas mal formuladas ${ }^{3-5}$.

Nesse sentido, advoga-se que, no processo de construção e gestão do campo da saúde, conforme os princípios e conceitos do Sistema Único de Saúde (SUS), o conhecimento gerado pelas pesquisas em saúde tem se constituído um instrumento importante para a tomada de decisão e um facilitador para seus agentes conhecerem e intervirem na produção e utilização de mais informações ${ }^{6}$. No bojo do contexto da pandemia de Covid-19, a ciência e a pesquisa 
estiveram em discussão e, algumas vezes, a temática protagonizou polarização. Para uns, o negacionismo se fez presente; para muitos, representou confiança e expectativa de respostas para o maior desafio do século. Afora essa situação que mais se configura como posicionamento político do que como valor atribuído à ciência, os autores deste artigo se posicionam como reconhecedores do potencial contributivo da ciência para o desenvolvimento humano e para o bem viver. Além disso, valorizam a produção científica para a tomada de decisão em saúde.

Pesquisadores, cientistas e organizações do mundo inteiro, a exemplo da Organização Mundial de Saúde (OMS), reforçam o papel da ciência no combate à Covid-19. Nesse ínterim, têm se mobilizado para estimar desde os efeitos da doença sobre a saúde da população, capacidade de diagnóstico da doença, estudo da patogênese - incluindo mecanismos de disseminação e transmissão viral -, desenvolvimento de materiais com ação antiviral, avaliação da atenção à saúde, prevenção dos impactos na população, até a análise dos impactos econômicos e sociais da pandemia no contexto mundial ${ }^{7-8}$.

Ainda com muitas interrogações sobre 0 conhecimento do comportamento da Covid-19, seu curso clínico, potenciais sequelas, reposicionamento de fármacos, formas de contágio, medidas preventivas agravadas pela ausência de uma vacina para prevenir ou tratar a infeção por SARS-CoV-2 (do inglês: Severe Acute Respiratory Syndrome Coronavirus 2), alinhadas a outros desdobramentos da doença e que impactam para além da saúde, a exemplo dos impactos econômicos, sociais e educacionais, a produção científica tem sido vista como ferramenta imprescindivel para respostas a estes vazios e investidas são feitas na busca da compreensão e soluções para a Covid-197-9. Corroborando o exposto, Núñez Novo et al. ${ }^{10}$ asseveram que, quando uma pandemia como a do novo coronavírus se alastra pelo mundo, uma das grandes necessidades é, obviamente, o conhecimento sobre a doença, assim como o desenvolvimento de terapias especificas para o tratamento ou até mesmo a produção de uma vacina.

A Constituição Federal do Brasil, promulgada em 1988, grafa a importância do desenvolvimento científico para o país, cabendo ao Estado, de acordo com os artigos 218 e 219, o dever de promover e incentivar a pesquisa e capacitação tecnológica, tendo em vista o bem público e a importância

\section{"Pesquisadores, cientistas \\ e organizações do mundo inteiro, a exemplo da OMS, reforçam o papel da ciência no combate à Covid-19"}

social que tal investimento inflige para o progresso tecnológico e para o crescimento econômico de um país ${ }^{11}$.

Sob essa égide, mas não restritas ao território nacional, diferentes frentes mundiais, particularmente protagonizadas por universidades e centros de pesquisas, têm investido na realização de estudos sobre a Covid-19. 0 desenvolvimento desses estudos tem contado com o apoio colaborativo de governos e de sistemas de saúde que anseiam por respostas a perguntas que orbitam cotidianamente 0 saber-fazer da gestão e atenção à saúde.

Diante do exposto, este artigo procura descrever duas pesquisas que tiveram como um dos campos de investigação, no curso da pandemia, o município de Sobral-CE e que colaboraram com subsídios para a indução de decisões em saúde.

\section{METODOLOGIA}

Trata-se de estudo exploratório descritivo, do tipo relato de experiência, com abordagem qualitativa, realizado no contexto de enfrentamento da pandemia de Covid-19 em Sobral, município localizado na zona norte do estado do Ceará, no período de junho a agosto de 2020.

Para Dyniewicz ${ }^{12}$, os relatos de experiência são metodologias de cunho observacional e com caráter sistemático da realidade, sem a necessidade de testar hipóteses; entretanto, estabelecendo as relações entre os achados dessa realidade e as bases teóricas pertinentes. Nesse tipo de abordagem, o fenômeno humano estudado é entendido como parte da realidade social, pois ele não se difere só por agir, mas por pensar sobre o que faz e interpretar suas ações dentro e a partir da realidade vivida e partilhada com seus semelhantes ${ }^{13}$.

0 sistema de saúde do município de Sobral é reconhecido em decorrência do modelo bem-sucedido e dos bons resultados alcançados nos diferentes 
setores e práticas da saúde. Formado por uma rede de atenção à saúde hierarquizada e regionalizada, com serviços em diferentes níveis de complexidade, é o polo de referência para a macrorregião norte do Ceará e é composto por 249 estabelecimentos e serviços relacionados à Atenção Primária, Atenção Especializada e Atenção Terciária. Tem, em seu ideário, a missão de "planejar e garantir a efetividade da política municipal de saúde, com excelência em todos os níveis de atenção à saúde, respeitando as diretrizes e princípios do SUS"14-15.

Destaca-se o fato de o município protagonizar a implantação de um modelo de organização e desenvolvimento de processos ensino, pesquisa e extensão no campo da formação em saúde, que paulatinamente se complexificou ao ponto de adquirir o status de Sistema Municipal Saúde Escola, instituído pela Lei municipal $n^{0} 1.685 / 2017^{16}$.

Em janeiro de 2020, antes do início da transmissão comunitária no Brasil e posterior ao status de pandemia de Covid-19 no mundo, Sobral teve um provável caso importado, posteriormente descartado. 0 surgimento prematuro do caso suspeito alertou a Secretaria Municipal de Saúde (SMS), que já no mês seguinte iniciou o planejamento e a reorganização da rede para o enfrentamento de uma possivel onda de casos de Covid-19. Com isso, foi disparada a elaboração do plano de contingência do município ${ }^{17-18}$.

Tão logo difundida a notícia da circulação do vírus no estado do Ceará, a Secretaria da Saúde de Sobral iniciou a adoção de medidas preventivas e protetivas para a população, orientada pelas recomendações da Organização Mundial da Saúde (OMS) e do Ministério da Saúde (MS). Por intermédio dos decretos $n^{\circ}$ $2.369 / 2020^{19}$ e $n^{0} 2.371 / 2020^{20}$, consecutivamente, foram anunciados estados de perigo público e emergência municipal. A partir de então, diversas medidas foram adotadas, dentre as quais podem ser citadas a adoção do isolamento social, da etiqueta respiratória, a proteção dos vulneráveis e grupos de riscos, o fechamento dos serviços de comércio e de ensino, a instituição de um comitê de crise e a intensificação do monitoramento e vigilância dos suspeitos, além da reorganização dos fluxos de atenção à saúde. Sobral teve o primeiro caso confirmado de Covid-19 em 17 de março de $2020^{21}$.

0 referido município participou, como campo de investigação, de duas pesquisas de cunho epidemiológico. Uma de âmbito nacional, intitulada: “Evolução e Prevalência de infecção por Covid-19

\section{"Ampliar o diálogo entre sociedade e comunidade científica para o uso eficiente da informação para a tomada de decisão em saúde"}

no Brasil", sob a coordenação de pesquisadores vinculados à Escola Superior de Educação Física da Universidade Federal de Pelotas (UFPel) ${ }^{22}$. E, outra, dirigida especificamente ao município de Sobral, promovida pela Secretaria de Saúde do Estado do Ceará (SESA) e Federação das Indústrias do Estado do Ceará (FIEC), sob a responsabilidade do Município de Sobral e do Instituto 0pnus, intitulada "Soroprevalência da Covid-19 no município SobralCE" 23 .

A participação da gestão municipal para a execução das pesquisas se deu na articulação, preparação, recepção da equipe de pesquisa e execução do campo de investigação, de modo que a narrativa histórica descrita se encontra fundamentada nas vivências de profissionais com gerência no campo da Atenção Primária, vigilância à saúde e pesquisa em saúde da gestão municipal de Sobral, traduzindo os aspectos de desenvolvimento das pesquisas, assim como as reflexões advindas e as transformações ocorridas ainda no curso da coleta das duas pesquisas.

\section{RESULTADOS E DISCUSSÃ 0}

Há várias questões passiveis de discussão na apresentação dos resultados da vivência das pesquisas “Evolução da prevalência de infecção por Covid-19 no Brasil: estudo de base populacional" e a pesquisa de "Soroprevalência de Covid-19 no município de Sobral". Contudo, neste texto será explorada a dinâmica do campo e o potencial contributivo dos resultados, ainda na fase de campo, na intenção de ampliar o diálogo entre sociedade e comunidade científica para o uso eficiente da informação para a tomada de decisão em saúde.

Nesse ínterim, o relato se encontra dividido em dois tópicos: "Reconhecimento da prevalência de Covid-19 como um subsídio para o planejamento em saúde" e "A pesquisa de soroprevalência da Covid-19: trajetórias de campo em Sobral". 
Reconhecimento da prevalência de Covid-19 como um subsidio para o planejamento em saúde

Sobral integrou o rol dos 133 municípios que constituíram o campo de investigação da pesquisa “Evolução da Prevalência de Infecção por Covid-19 no Brasil: estudo de base populacional". Pesquisa inicialmente financiada pelo Ministério da Saúde, coordenada pela Universidade Federal de Pelotas, do Rio Grande do Sul, e executada pelo Instituto Brasileiro de Opinião Pública e Estatística (Ibope). Contou, no curso de seu desenvolvimento, com o financiamento complementar do Programa Todos Pela Saúde, fundo criado pelo Itaú Unibanco para apoiar o enfrentamento da Covid-19 em diversas frentes, entre elas, o suporte às iniciativas de pesquisa científica ${ }^{24}$. Apresentava como objetivo central estimar a prevalência de indivíduos infectados por SARS-CoV-2 no Brasil; subsidiariamente, visava a avaliar sintomas e analisar a velocidade de expansão da infecção em uma amostra significativa da população brasileira. Planejada, a princípio, para realizar três inquéritos epidemiológicos, no itinerário da pesquisa foi acrescida mais uma fase, totalizando quatro inquéritos transversais com amostragens em municípios sentinelas ${ }^{22}$.

Registra-se que a definição dos municípios sentinelas se deu com base na exiguidade do tempo e na disponibilidade limitada de testes no período da pesquisa. A identificação dos municípios sentinela seguiu os parâmetros do Instituto Brasileiro de Geografia e Estatística (IBGE) e elegeu, em cada estado federativo, a área urbana de municípios sede de cada sub-região de saúde. Em todos os 133 municípios foram selecionados aleatoriamente domicílios em setores censitários ${ }^{22}$.

Ao tomar conhecimento da eleição de Sobral como um dos municípios a integrar o campo de investigação, a gestão municipal, com o seu dispositivo de ordenamento da pesquisa, a Escola de Saúde Pública Visconde Saboia, realizou análise tácita e compreendeu a importância e o potencial contributivo que a pesquisa traria para o melhor reconhecimento epidemiológico da Covid-19 no município e o planejamento de ações relacionadas, haja vista os benefícios que as evidências científicas podem proporcionar ao processo de tomada de decisão nas políticas de saúde ${ }^{25}$. Ademais, o delineamento metodológico do estudo, com sucessivos inquéritos e com a seleção da amostragem de forma aleatória,

\section{"Retorno da pesquisa aos serviços de saúde e ao bem-estar social, já no curso da coleta de dados"}

permitiria prospectivamente uma estimativa da prevalência com a possibilidade de captura de casos assintomáticos e, dessa forma, um agir na interrupção da cadeia de transmissão.

A realização da pesquisa ocorreu no período de maio a agosto de 2020, tendo sido abordados 250 participantes, com a realização de entrevistas e teste sanguíneo rápido para Covid-19. Em Sobral, o campo foi iniciado em 14 de maio. Em todo o território nacional, foi observada a insuficiência de diálogos interinstitucionais entre os proponentes da pesquisa e os campos de investigação, ocasionando, em muitos municípios, a princípio, a não adesão dos gestores e/ou da população à realização da coleta de dados na fase inicial. Sobral não vivenciou essas ocorrências, visto que, conforme já registrado, agiu com rapidez e protagonizou o diálogo com as instituições responsáveis buscando esclarecimentos, tendo em vista que também não fora contatado antecipadamente para planejamento do campo. No entanto, no curso do primeiro dia, na concomitância do início da coleta de dados, já com os entrevistadores nos territórios pré-selecionados, foram identificados alguns percalços rapidamente contornados, a partir do esclarecimento das instituições sobre a legitimidade da pesquisa, seu itinerário metodológico e as contrapartidas requeridas.

Já no exercício do primeiro inquérito, uma solicitação do município de Sobral, que foi incorporada pela coordenação geral da pesquisa, foi a de repasse diário dos casos positivos identificados para o comitê de crise municipal para que esse pudesse intervir epidemiologicamente com o acompanhamento dos casos e de seus contatos. Essa decisão foi de suma relevância, pois permitiu o retorno da pesquisa aos serviços de saúde e ao bem-estar social, já no curso da coleta de dados. Além disso, dada a magnitude do problema da Covid-19, reconhecer os casos de sintomáticos leves e assintomáticos é um passo estratégico para o desenvolvimento de decisões assertivas, visto 


\section{"A busca ativa de sintomáticos respiratórios representa a estratégia inicial para a detecção de casos novos e a primeira dentre as várias ações de controle"}

que esses precisam ser acompanhados, pois podem transmitir o vírus e ampliar o número de $\operatorname{casos}^{26}$.

As lições aprendidas na execução do primeiro inquérito fizeram com que a coordenação nacional, em cooperação com as Secretarias Municipais de Saúde, identificasse um profissional local, de preferência vinculado à área da vigilância sanitária, para acompanhar a execução da pesquisa. Nesse contexto, Sobral prontamente designou a gerente da célula da vigilância sanitária para tal fim. Essa profissional, uma enfermeira, passou a dar suporte técnico aos entrevistadores no campo, estabelecer contato direto com a Coordenação da Atenção Primária para que essa pudesse difundir entre as equipes da Estratégia Saúde da Família (ESF) a agenda da pesquisa e a continuidade do apoio necessário para a sua execução; bem como dar continuidade à socialização dos casos identificados ao Escritório de Crise para a tomada de decisão.

Destaca-se a boa comunicação e corresponsabilidade entre a gestão da pesquisa e da saúde de Sobral. Os resultados diários do campo eram encaminhados ao comitê de crise, em formato de relatório. Nos relatórios, constavam as informações sobre o participante com teste positivo (nome, idade e endereço) e seus contatos testados. Essas informações eram desagregadas e socializadas para os territórios com casos identificados. Após o recebimento das informações, a Coordenação da Atenção Primária as direcionava para cada Centro de Saúde da Família (CSF) de referência, disparando, assim, a identificação do caso/busca ativa, monitoramento e rastreio dos contatos e, por conseguinte, desencadeando ações de prevenção da disseminação do vírus.

A busca ativa de sintomáticos respiratórios representa a estratégia inicial para a detecção de casos novos e a primeira dentre as várias ações de controle da doença no território, sendo incorporada ao processo de trabalho das equipes da ESF. A fim de concretizar o cuidado de sintomáticos suspeitos ou confirmados para o SARS-CoV-2, foram implementadas estratégias de vigilância e monitoramento desses casos no município de Sobral, com base em protocolos e diretrizes nacionais e estaduais. 0 exposto corrobora o reconhecimento de que compete à Atenção Primária à Saúde (APS), por meio da Estratégia Saúde da Família, realizar a identificação dos casos de Covid-19, a classificação da gravidade desses, direcionando o cuidado aos pacientes nos demais pontos de atenção à saúde ${ }^{27}$, quando necessário.

Ademais, embora para os casos mais leves seja indicado o isolamento domiciliar, isso não exime a atuação da APS no monitoramento e acompanhamento clínico até a alta. E, para os casos graves, incluise a estabilização clínica e o encaminhamento para outros serviços da Rede de Atenção à Saúde (RAS). Para tanto, a APS deve assumir um papel resolutivo frente às situações classificadas como mais leves, além da identificação precoce e o encaminhamento rápido e correto dos casos mais graves, mantendo a coordenação do cuidado desses últimos ${ }^{28}$.

Outro aspecto importante advindo da participação do município nesse estudo foi a superação de alguns desafios como a subnotificação de casos, considerando a existência de casos assintomáticos. Outrossim, essa identificação de novos casos colaborou também para a aferição de territórios e/ou grupos mais vulneráveis. Dessa maneira, as informações obtidas pela pesquisa auxiliaram o traçar de estratégias, principalmente a ampliação do rastreamento, considerando outras medidas já incorporadas pela gestão da saúde do município no monitoramento dos casos suspeitos e confirmados de Covid-19 pela equipe de Saúde da Família (eSF), conforme os critérios de risco. Para grupos considerados prioritários, como gestantes, idosos, crianças menores de cinco anos, profissionais de saúde e profissionais da segurança, o monitoramento acontecia diariamente, inclusive nos finais de semana e feriados; nos outros casos, em dias alternados.

Tais ações são coerentes com o preconizado pelo MS na medida em que o monitoramento envolve diversas formas de cuidado pela equipe interdisciplinar, respeitando-se a singularidade do paciente e/ou da família. Dentre algumas dessas ações, incluem-se o acolhimento, o atendimento individual, a teleconsulta, os teleatendimentos e as visitas domiciliares, todas consideradas ferramentas potentes que vêm sendo utilizadas para o cuidado, a 


\section{"Gestão municipal de Sobral se ancorou fortemente nas evidências científicas produzidas para embasar tomadas de decisão"}

garantia da segurança do paciente e a resolubilidade das demandas atendidas ${ }^{1-2}$. Nessa perspectiva, para atender às necessidades que são emanadas pela Covid-19, reafirma-se a necessidade de uma APS que recupere seus princípios de base comunitária, trabalho em equipe, intra e extramuros, com usuários, pacientes, famílias e comunidade, visando à integralidade e à humanidade das ações em toda a linha de cuidado ${ }^{27}$.

Por fim, no que concerne ao desenvolvimento da pesquisa de base populacional, "Evolução da prevalência de infecção por Covid-19 no Brasil", registra-se que um outro desafio dos serviços de saúde é a destinação adequada de resíduos sólidos. Nesse sentido, o município de Sobral viabilizou o recebimento dos resíduos gerados no processo de coleta de dados da pesquisa. A recepção desses elementos foi atribuição dos CSF, que já dispõem de protocolos e fluxos estabelecidos para tal e, no período noturno e finais de semana, ficou sob encargo do hospital geral que estava sob a administração municipal. 0 lixo infectante continha os Equipamentos de Proteção Individual (EPIs) utilizados e demais materiais usados pelos entrevistadores, assim como as caixas de acondicionamento de material perfurocortante utilizado durante a coleta de sangue para a realização dos testes rápidos.

0 comprometimento em realizar o gerenciamento de resíduos por parte dos municípios visa, fundamentalmente, à saúde humana, animal e ao meio ambiente. 0 s resíduos gerados na realização da pesquisa, de acordo com a classificação da Agência Nacional de Vigilância Sanitária (Anvisa), são dos tipos $A$ e E e exigem uma segregação, acondicionamento, identificação, transporte, destinação e disposição final ambientalmente adequados, observando as normas operacionais específicas, de modo a evitar danos ou riscos à saúde pública e à segurança e a minimizar os impactos ambientais adversos ${ }^{29}$.

Entende-se que a aplicação do conhecimento produzido pela pesquisa científica, a disponibilidade de informações atualizadas e fidedignas e a observância dos marcos legais existentes foram diretrizes observadas no desenvolvimento e incorporação de resultados oriundos dessa pesquisa. 0 exposto corrobora a análise realizada por Silva, Sousa e Barreto ${ }^{25}$ a referirem o papel da pesquisa. Esses autores acrescentam ainda que as pesquisas necessitam ser amplamente disseminadas para tornar os serviços e sistemas de saúde mais efetivos e sustentáveis.

\section{A pesquisa de soroprevalência da Covid-19: trajetórias do campo em Sobral}

A saúde é um campo fértil para as inovações informadas por evidências. Isso se faz palpável a partir do apoio às experiências inovadoras, da confiança na recombinação de ideias no âmbito da implementação das ações e do incentivo de ambientes institucionais favoráveis à mudança ${ }^{25}$.

Nesse sentido, alguns esforços têm sido implementados para que as políticas, sistemas e serviços de saúde sejam mais informados pelas melhores evidências, conhecimentos e valores sociais existentes ${ }^{30}$. De modo que, no contexto da pandemia, a gestão municipal de Sobral se ancorou fortemente nas evidências científicas produzidas para embasar tomadas de decisão.

A pesquisa de soroprevalência da Covid-19, promovida pelo Governo do Ceará, por meio da Secretaria de Saúde do Estado, em parceria com a Prefeitura Municipal de Sobral e o Instituto Opnus, para fins de enfrentamento da emergência de saúde pública de importância internacional decorrente do novo coronavírus ${ }^{23}$, foi uma, dentre as múltiplas estratégias de boas práticas em gestão, diante da pandemia em curso.

Ela tinha como objetivo central avaliar e ampliar - controle epidemiológico, bem como orientar as políticas públicas de prevenção e combate à Covid-19. Esses objetivos buscaram estimar 0 percentual de pessoas com anticorpos para o SARS-CoV-2, o percentual da infecção em pessoas assintomáticas ou subclínicas, obter cálculos mais precisos da letalidade da doença e analisar a velocidade de expansão da infecção ao longo do tempo.

Um diferencial em relação à pesquisa nacional, apresentada na seção anterior, é que essa pesquisa de soroprevalência teve seu desenvolvimento sob total gerência do município. Desse modo, a pesquisa foi realizada em duas fases, nos meses de junho a agosto 
de 2020. Foram avaliadas a transmissibilidade e a evolução do vírus em diferentes períodos de tempo. Seu percurso metodológico orientava a condução de sorteio aleatório, semelhante à utilizada no censo populacional. Foram sorteados 40 setores dentro dos territórios da ESF de Sobral para a testagem da população.

A equipe foi composta por uma supervisora de campo, assistentes de pesquisa (estudantes de cursos de graduação de Enfermagem e Biomedicina), além de profissionais da eSF, que foram capacitados a fim de estarem munidos de todas as orientações necessárias para a execução do campo, de modo a garantir confiabilidade ética e a estarem cientes dos procedimentos de biossegurança necessários para o campo e evitar qualquer risco de viés possível. Em ambas as fases, a partir das ruas sorteadas, a equipe de pesquisa, orientada por uma supervisora de campo, chegava ao domicílio e, inicialmente, apresentavase e informava sobre a pesquisa, ressaltando sua importância e a relevância social para o município. Em seguida, era realizado, de forma aleatória, o sorteio de quem do domicílio passaria pela testagem. A intenção da estratégia adotada visava garantir representatividade estatística da população no que refere ao sexo e idade. De posse da identificação do domiciliado sorteado, era realizada a testagem seguindo todo o protocolo de cuidado, assim como era aplicado um inquérito de dados socioeconômicos.

Em cada participante foi realizado o teste rápido, utilizado para a detecção de anticorpos IgM/IgG contra SARS-CoV-2, e o RT-PCR (swab), que é um teste de biologia molecular capaz de detectar a presença do vírus no paciente. Tal procedimento permite perceber como o vírus age nos pacientes sintomáticos e assintomáticos - que não apresentam sintomas de Covid-19. Em cada uma das etapas, 800 pessoas foram testadas, totalizando 1.600 exames. Além da realização dos testes, foi aplicado um questionário epidemiológico e social com informações sobre sexo, idade, escolaridade, condições de saúde e possíveis sintomas apresentados.

Destaca-se a importante mobilização e o papel das eSF que compõem a ESF do município de Sobral. Diversos profissionais contribuíram no apoio e execução do campo, com a participação ativa de 15 enfermeiros, 15 dentistas, seis profissionais do Núcleo Ampliado de Saúde da Família e Atenção Básica (Nasf-AB) e 416 agentes comunitários de saúde (ACS), além dos dez supervisores de campo, vinculados ao Instituto 0pnus, e 67 estudantes

\section{"A boa comunicação existente entre a ESF e a comunidade possibilita a garantia do pleno desenvolvimento de estudos e ações"}

de graduação em Enfermagem e Biotecnologia, pertencentes às instituições de Ensino Superior situadas em Sobral, que atuaram na realização dos exames e orientações de prevenção e cuidados com a Covid-19.

Cabe realçar o importante papel desempenhado pelos ACS nas orientações para a adesão dos participantes à pesquisa. Isso se reflete no forte vínculo que esses trabalhadores possuem com a comunidade adscrita em seus territórios de atuação. A boa comunicação existente entre a ESF e a comunidade possibilita a garantia do pleno desenvolvimento de estudos e ações, uma vez que conhecem a densidade e a particularidade dos territórios de atuação. Para além do vínculo prévio, outro fator que contribuiu para a adesão foi a identificação dos pesquisadores de campo, que portavam crachás de sinalização da pesquisa ${ }^{31}$.

Um dado importante advindo dessa articulação entre a equipe de pesquisa e a ESF foi, ainda no curso da execução da pesquisa, a socialização das informações obtidas com a gestão municipal, permitindo auxiliar de forma satisfatória 0 monitoramento e a vigilância dos pacientes com Covid-19. Ao serem testados e diagnosticados como casos positivos, os participantes eram orientados quanto aos protocolos de isolamento domiciliar, monitorados diariamente pela eSF de referência por meio de ligações telefônicas e/ou mensagens de textos, respeitando as singularidades dos perfis. Ademais, visitas domiciliares eram realizadas diariamente para a melhor condução e acompanhamento clínico de cada paciente.

Findadas as etapas e o tratamento dos dados, com a posterior divulgação dos relatórios com os resultados de cada uma das fases da pesquisa pela SESA, foi possível observar uma redução significativa do número de pessoas com o vírus ativo adscritas a Sobral. Tais resultados demonstraram a eficácia das ações de combate à Covid-19 adotadas 
pelo município de Sobral no referido período e subsidiaram as autoridades sanitárias e o governo municipal no planejamento de retomada gradativa de atividades socioeconômicas dessa região, na organização de novas estratégias de rastreamento de contatos, no monitoramento dos pacientes com Covid-19, na potencialização de ações de prevenção com foco em áreas com maior concentração de casos positivos, entre outras ações. Contudo, reconhecese que o alcance dos resultados apresentados se deve a uma teia complexa e bem entrelaçada de decisões políticas, tácitas, técnicas, intersetoriais, interprofissionais que, orientadas por uma gestão comprometida com o bem maior e a vida, ultrapassaram barreiras e investiram fortemente para mitigar os impactos da pandemia. E, dentre esses movimentos, esteve presente a pesquisa como orientadora de boas práticas em saúde.

\section{CONCLUSÃO}

As experiências relatadas caracterizam a relevância e as contribuições da ciência, por meio do desenvolvimento de pesquisas, para a produção de conhecimento e auxílio à gestão em saúde na tomada de decisão embasada. 0 êxito desse processo se deve, em grande parte, à postura dialógica das equipes das pesquisas e da gestão local, de modo a garantir a consecução dos objetivos propostos e a superar desafios inerentes ao campo e agravados pelo contexto sanitário da pandemia.

É significativo destacar o papel da pesquisa de campo, em particular no contexto aqui relatado, como importante instrumento de reconhecimento e mensuração do fenômeno, haja vista que permite, com fidedignidade, reconhecer a situação epidemiológica do município e inferir seus processos de determinação.

Ademais, por se tratar a Covid-19 de uma situação epidemiológica ainda recente, com insuficiência de informações, sugere-se que novas pesquisas sejam realizadas, atentando para os atuais vazios do conhecimento, incluindo o curso progressivo da doença. Outrossim, é ensejado que a gestão em saúde possa ser referenciada na incorporação de evidências científicas para ampliar a efetividade das ações.

\section{CONTRIBUIÇÃO DAS AUTORAS}

Maria Socorro de Araújo Dias contribuiu com redação, análise e revisão crítica do manuscrito.
Verena Emmanuelle Soares Ferreira contribuiu com redação, análise e revisão crítica do manuscrito. Rogeriany Lopes Farias contribuiu com redação, análise e revisão crítica do manuscrito. Lielma Carla Chagas da Silva contribuiu com redação, análise e revisão crítica do manuscrito. Heliandra Linhares Aragão contribuiu com redação, análise e revisão crítica do manuscrito. Rafaela Costa Porto contribuiu com redação, análise e revisão crítica do manuscrito.

\section{REFERÊNCIAS}

1. Oliveira AG, Silveira D. A importância da ciência para a sociedade - editorial. Infarma Ciências Farmacêuticas [serial on the internet]. 2013 [cited 2020 0ct 31];25(4):169. Available from: http:// revistas.cff.org.br/?journal $=$ infarma $\&$ page $=$ article \&op $=$ view \&path $\% 5 B \% 5 D=572 \&$ path $\% 5 B \% 5 D=p d f$

2. Branco MAF. Informação e saúde: uma ciência e suas políticas em uma nova era. Rio de Janeiro: Ed. Fiocruz; 2006.

3. Barreto J0M, Souza NM. Making progress in the use of health policies and practices informed by evidence: the Piripiri-Piaui experience. Cien Saude Colet [serial on the internet]. 2013 [cited $20200 \mathrm{ct}$ 31];18(1):10. Available from: https://www.scielo. $\underline{\mathrm{br} / \mathrm{pdf} / \mathrm{csc} / \mathrm{v} 18 \mathrm{n} 1 / 04 . \mathrm{pdf}}$

4. Head BW. Toward More Evidence-Informed Policy Making? Public Adm Rev [serial on the internet]. 2016 [cited 2020 Nov 02];76(3):47284. Available from: https://onlinelibrary.wiley. com/doi/epdf/10.1111/puar.12475

5. Langlois EV, Montekio VB, Young T, Song K, Alcalde-Rabanal J, Tran N. Enhancing evidence informed policymaking in complex health systems: lessons from multi-site collaborative approaches. Heal Res Policy Syst [serial on the internet]. 2016 [cited 2020 Nov 02];14(1):20. Available from: https://www.ncbi.nlm.nih.gov/ $\mathrm{pmc/articles/PMC4794922/pdf/129612016}$ Article 89.pdf

6. Patriota CMM. 0 uso da informação em saúde para tomada de decisão: um estudo de metanálise [dissertação]. Rio de Janeiro: Fundação Oswald Cruz; 2009.

7. Kohls C. Especialistas reforçam papel da ciência no combate ao novo coronavírus. Superintendência de Comunicação e Marketing (Sucom) e Agência Escola de Comunicação Pública e Divulgação Científica e Cultural da UFPR [serial on the internet]. 2020 [cited 2020 0ct 26]. Available from: https://www. ufpr.br/portalufpr/noticias/especialistas-reforcampapel-da-ciencia-no-combate-ao-novo-coronavirus/ 
8. Negri F, Zucoloto G, Miranda P, Koeller P. Ciência e Tecnologia frente à pandemia: como a pesquisa científica e a inovação estão ajudando a combater o novo coronavírus no Brasil e no mundo [document on the internet]. Brasília (DF): Ipea; 2020 [cited 2020 Nov 02]. Available from: https://www.ipea.gov.br/ cts/pt/central-de-conteudo/artigos/artigos/182corona

9. Brasil. Boletim epidemiológico $n^{0} 7$ - Doença pelo coronavírus [document on the internet]. Brasília (DF): Ministério da Saúde; 2020 [cited 2020 Nov 02]. Available from: https://www.saude. gov.br/images/pdf/2020/April/06/2020-04-06-BE7Boletim-Especialdo-COE-Atualizacao-da-Avaliacaode-Risco.pdf

10. Núñez Novo B. A importância da ciência, tecnologia e inovação em tempos de pandemia para a sociedade. Brasil Escola - meu artigo [serial on the internet]. 2020 [cited 2020 0ct 28]. Available from: https://meuartigo.brasilescola.uol.com.br/direito/ a-importancia-da-ciencia-tecnologia-e-inovacaoem-tempos-de-pandemia-para-a-sociedade.htm

11. Brasil. Constituição da República Federativa do Brasil de 1988. Brasília (DF): Senado Federal; 1988.

12. Dyniewicz AM. Metodologia da pesquisa em saúde para iniciantes. 2. ed. São Caetano do Sul: Difusão; 2009.

13. Minayo MCS. 0 desafio do conhecimento: pesquisa qualitativa em saúde. São Paulo: Hucitec; 2007.

14. Brasil. Distribuição dos estabelecimentos de saúde de Sobral - 2019 [document on the internet]. Brasília (DF): Ministério da Saúde; 2019 [cited 2020 Nov 10]. Available from: http://cnes.datasus. gov.br/pages/estabelecimentos/consulta.jsp

15. Sobral. Escola de Saúde Pública Visconde de Saboia. Plano Municipal de Educação Permanente em Saúde de Sobral-CE 2019-2021. Sobral (CE): Secretaria Municipal da Saúde de Sobral; 2019.

16. Sobral. Lei $\mathrm{n}^{\circ} 1685$ de 08 de novembro de 2017. Institui, no âmbito do município de Sobral, - Sistema Municipal Saúde Escola [document on the internet]. Diário Oficial do Município, Sobral (CE); (2017 Nov 10 [cited 2020 Nov 10]). Available from: http://transparencia.sobral.ce.gov.br Larquivo/nome:f6339d39de984d3e915eff696a6f 4f52.pdf

17. Sobral. Prefeitura Municipal de Sobral. Decreto $n^{\circ} 2370$, de 13 de março de 2020. Institui o plano municipal de contingência para o coronavírus - covid 19 [document on the internet]. Diário Oficial do Município, Sobral (CE); (2020 Mar 20 [cited 2020 Nov 11]). Available from: http://transparencia.sobral. ce.gov.br/arquivo/nome:352d4d59debb8f77678c8e1 d3c50cac6.pdf
18. Ribeiro MA, Araújo Junior DG, Cavalcante ASP, Martins AF, Sousa LA, Carvalho RC, et al. (RE) Organização da Atenção Primária à Saúde para o enfrentamento da COVID-19: Experiência de SobralCE. APS em Revista [serial on the internet]. 2020 [cited 2020 Nov 15];2(2):177-88. Available from: https://apsemrevista.org/aps/article/view/125/54

19. Sobral. Decreto $n^{0} 2.369$ de 13 de março de 2020. Declara estado perigo público iminente. Diário Oficial do Município. Sobral (CE); 2020.

20.Sobral. Prefeitura Municipal de Sobral. Decreto $\mathrm{n}^{\circ} 2.371$, de 16 de março de 2020. Decreta estado de emergência no âmbito do município de sobral e estabelece medidas para enfrentamento do novo coronavírus (covid-19) [document on the internet]. Diário Oficial do Município, Sobral (CE); (2020 Mar 16 [cited 2020 Nov 09]). Available from: http://www.sobral.ce.gov.br/diario/ public/files/diario/9bf7ba4ae2931bdf14b3a48b524 $\underline{0 \text { d141.pdf }}$

21. Sobral. Boletim epidemiológico [document on the internet]. Sobral (CE): Prefeitura Municipal de Sobral. 2020 [cited 2020 Nov 09]. Available from: http://www.sobral.ce.gov.br/informes/ principais/boletim-covid-19-em-sobral

22. Universidade Federal de Pelotas. Evolução da prevalência de infecção por Covid-19 no Brasil: estudo de base populacional. Portal Institucional [document on the internet]. 2020 [cited 2020 Nov 02]. Available from: https://institucional.ufpel. edu.br/projetos/id/u2620

23. Ceará. Iniciada segunda fase da Pesquisa de Soroprevalência em Sobral [document on the internet]. Ceará: Governo do Estado; 2020 [cited 2020 Nov 02]. Available from: https://www.ceara. gov.br/2020/08/04/iniciada-segunda-fase-dapesquisa-de-soroprevalencia-em-sobral/

24. Conselho Nacional de Secretarias Municipais de Saúde. Epicovid19 anuncia próxima etapa da pesquisa nacional [document on the internet]. 2020 [cited 2020 Nov 12]. Available from: https:// www.conasems.org.br/epicovid19-anuncia-proximaetapa-da-pesquisa-nacional-sobre-coronavirus/

25. Silva SF, Souza NM, Barreto J0M. Fronteiras da autonomia da gestão local de saúde: inovação, criatividade e tomada de decisão informada por evidências. Cien Saude Colet [serial on the internet]. 2014 [cited 2020 Nov 09];19(11):442738. Available from: https://www.scielo.br/ $\mathrm{pdf} / \mathrm{csc} / \mathrm{v} 19 \mathrm{n} 11 / 1413-8123-\mathrm{csc}-19-11-4427 . \mathrm{pdf}$

26. Hallal PC, Horta BL, Barros AJD, Dellagostin $0 A$, Hartwig FP, Pellanda LC, et al. Evolução da prevalência de infecção por COVID-19 no Rio Grande do Sul, Brasil: inquéritos sorológicos seriados. Cien Saude Colet [serial on the internet]. 2020 [cited 2020 Nov 12];25(Suppl 1):2395- 
2401. Available from: http://www.scielo.br/ scielo.php? script $=$ sci arttext $\&$ pid $=S 1413-812320$ $20006702395 \& \operatorname{lng}=$ en

27. Neto FRGX, Araújo CRC, Silva RCC, Aguiar $M R$, Sousa LA, Serafim TF, et al. Coordenação do Cuidado, vigilância e monitoramento de casos de Covid19 na Atenção Primaria à Saúde. Rev Enferm foco (Brasilia) [serial on the internet]. 2020 [cited 2020 Nov 12];11(1):[about 7 p.]. Available from: http://revista.cofen.gov.br/index.php/enfermagem/ article/view/3682/835

28. Brasil. Protocolo de Manejo Clínico do Coronavírus (Covid-19) na Atenção Primária à Saúde. Brasília (DF): Ministério da Saúde; 2020.

29. Brasil. Resolução. RDC $n^{0} 222$, de 28 de março de 2018. Regulamenta as Boas Práticas de Gerenciamento dos Resíduos de Serviços de Saúde e dá outras providências [document on the internet]. Brasília: Agência Nacional de Vigilância Sanitária; 2018 [cited 2020 Nov 03]. Available from: https:// www.gov.br/anvisa/pt-br

30. World Health Organization. World Health Report 2012: No Health Without Research. [document on the internet]. 2011 [cited 2020 Nov 10]. Available from: http://www.who.int/ rpc/whr2012/en/index.html

31. Ceará. Pesquisa epidemiológica que avalia impacto do coronavírus será realizada em Sobral [document on the internet]. Ceará: Governo do Estado; 2020 [cited 2020 Nov 05]. Available from: https://www.saude.ce.gov.br/2020/06/17/pesquisaepidemiologica-que-avalia-impacto-do-coronavirussera-realizada-em-sobral/
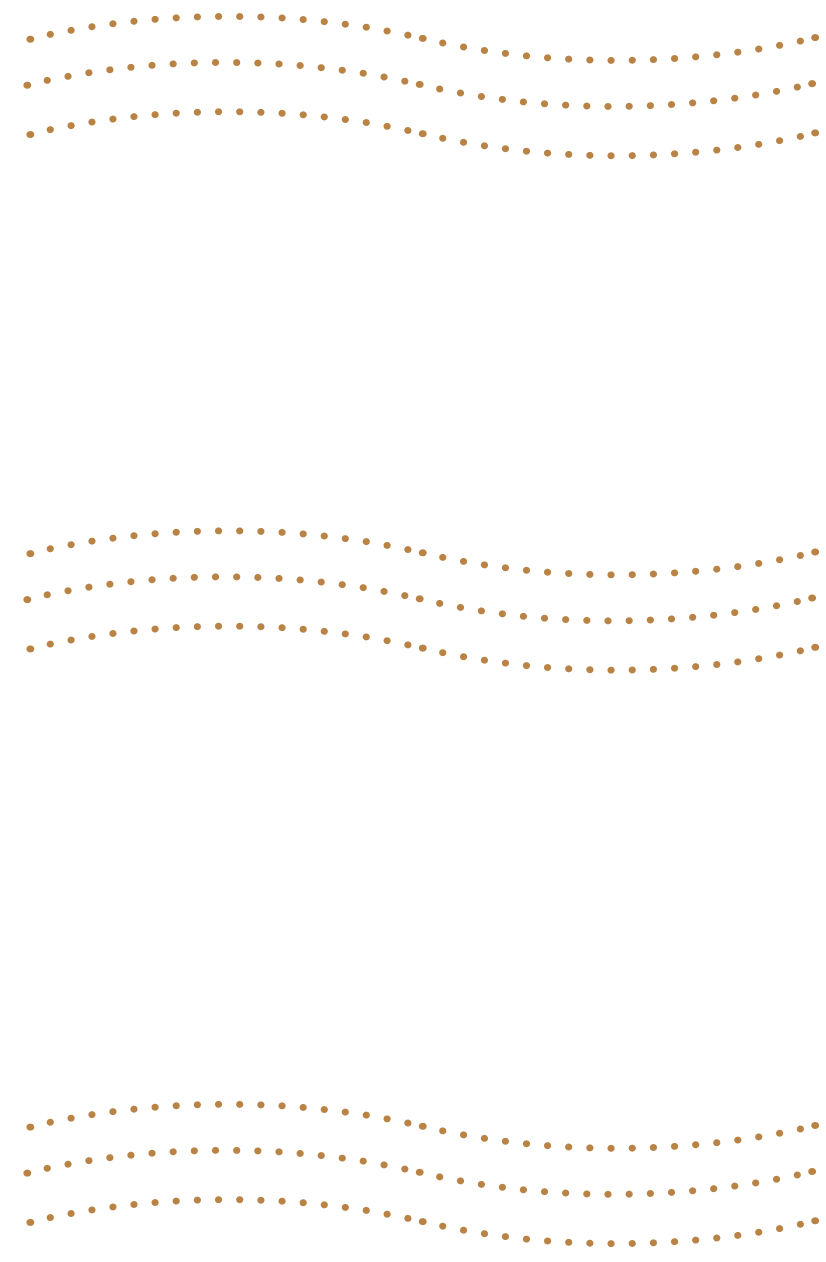